\title{
Sepsis induced coagulopathy score and D-dimer levels in COVID-19 patients followed in intensive care; what has changed in COVID era?
}

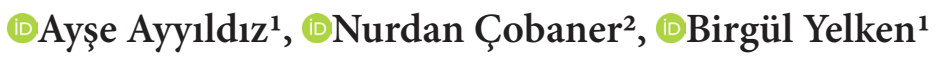 \\ ${ }^{1}$ Osmangazi University, Faculty of Medicine, Department of Anesthesiology and Reanimation, Eskişehir, Turkey \\ ${ }^{2}$ Van Training and Research Hospital, Intensive Care Clinic, Van, Turkey
}

Cite this article as: Ayyıldız A, Çobaner N, Yelken B. Sepsis induced coagulopathy score and D-dimer levels in COVID-19 patients followed in intensive care; what has changed in COVID era? J Health Sci Med 2022; 5(1): 94-98.

\begin{abstract}
Aim: This study was planned to compare the extent of hypercoagulopathic complications in COVID-19 pneumonia with that of last year's pneumonia cases which consist of by other agents.

Material and Method: The data of patients with pneumosepsis due to non-COVID-19 causes between 01 April-30 June 2019 and COVID-19 pneumosepsis patients between 01 April-30 June 2020,were analyzed retrospectively. Demographic data, comorbidities, SOFA scores, SIC scores, D-dimer levels, coagulopathic complications, mortality and discharge status of patients diagnosed with pneumosepsis and treated in both periods were recorded

Results: While sequential D-dimer measurements did not show a significant change in the 2019 group, it was observed that it increased significantly in the 2020 group $(\mathrm{p}<0.05)$. When we analyse the coagulopathic complications, we saw that submassive pulmonary embolism was recorded in one patient in the 2019 group.In the 2020 group, one patient had disseminated intravascular coagulation (DIC) and one patient had massive pulmonary embolism.

Conclusion: In our study, we did not find a significant difference in SIC scores and mortality rates between pneumosepsis patients in last years and COVID-19 pneumosepsis patients.
\end{abstract}

Keywords: COVID-19, coagulopathy, sepsis

\section{INTRODUCTION}

SARS-CoV-2 is a member of the beta coronavirus family and is the third coronavirus identified in the pandemic type (1). The potential for multiple organ damage is quite high, and these patients should be evaluated as multisystemic (2). With current literature, it has been observed that coagulopathic complications can frequently occur in COVID-19 infection. The mechanisms of these coagulopathic complications are not yet understood and many hypotheses have been proposed (3).

Similar to SARS-CoV, SARS-CoV-2 can enter host cells and directly damage endothelial cells via the angiotensin converting enzyme-2 (ACE-2) receptor. The existing comorbidities of the patients, immobility, venous stasis and high inflammation secondary to sepsis can be listed as other causes of coagulopathy. Activation of host defense systems (activation of humoral and cellular immunity) in sepsis gives rise to the concept called thromboinflammation or immunotrombosis (4). During the activation of humoral and cellular immunity pathways, increased inflammatory mediators cause platelet aggregation, peripheral vasoconstriction resulting from increased thromboxane production and endothelial dysfunction. Thrombin, which is formed by these mechanisms, increases the risk of coagulation (5).

COVID-19 coagulopathy can occur in different coagulopathic forms such as sepsis induced coagulopathy (SIC), thrombotic microangiopathy, disseminated intravascular coagulation (DIC), antiphospholipid syndrome, hemophocytic syndrome (6). Shock and development of DIC are two important causes of multiple organ damage in sepsis patients. DIC development in patients is an important predictor of mortality. Since early diagnosis is vital, it should be followed closely $(7,8)$. Sepsis-induced coagulopathy scoring is a scoring system 
created by adding platelet count and INR values to the Sequential Organ Failure Assessment (SOFA) scoring data. It is the first scoring system specially designed for coagulation disorders in sepsis after sepsis-3 definitions. In the early diagnosis and treatment of coagulopathic pathologies, regular follow-up of these scoring systems is recommended to reduce mortality (9).

This study was planned to compare the extent of these hypercoagulopathic complications in COVID-19 pneumosepsis worldwide with the scores of pneumosepsis cases that occurred with other pneumosepsis agents last year.

\section{MATERIAL AND METHOD}

The study was carried out with the permission of Eskişehir Osmangazi University Faculty of Medicine Non-Interventional Clinical Researchs Ethics Committee (Date: 16.10.2020, Decision No: 14). All procedures were carried out in accordance with the ethical rules and the principles of the Declaration of Helsinki.

In our study, the data of pneumosepsis patients with different etiological agent between 01 April-30 June 2019 and the data of COVID-19 pneumosepsis patients between 01 April-30 June 2020 were analyzed retrospectively. In the 2019 group, the number of patients followed in the intensive care unit was 92 , and 24 patients had a diagnosis of pneumosepsis. In the 2020 group, the number of patients followed in the intensive care unit was 145 , and 57 patients were diagnosed with pneumosepsis. The number of patients with positive COVID-19 PCR test results is thirty-two. Patients with a previous history of deep venous thrombosis in both groups were excluded from the study. SOFA score and SIC score were calculated every other day for the patients during their stay in the intensive care unit. D-dimer levels were followed consequtive as well as SOFA and SIC scoring. Values above $0.5 \mathrm{mg} / \mathrm{L}$ were accepted as the upper limit. Four patients in 2019 group and 2 patients in 2020 group were excluded from the study because they did not have a 3 consecutively calculated SIC score. Fifty patients' data were analyzed. Thrombosis prophylaxis was applied to all patients according to the recommendations of the International Society for Thrombosis and Hemostasis (ISTH). Low molecular weight heparins (LMWH) are routinely used for prophylaxis. Heparin therapy was initiated in patients with low renal clearance. When a thromboembolic complication developed, the dose of LMWH was increased by $25 \%$ and the treatment dose was increased. Demographic data, comorbidities, SOFA scores, SIC scores, D-dimer levels, coagulopathic complications, mortality and discharge status of patients diagnosed with pneumosepsis and treated in both periods were recorded.

\section{Statistical Analysis}

The analysis of the data was made with the SPSS-25 program and it was worked with a 95\% confidence level. Frequency (n) and percentage (\%) for categorical variables; Mean (X) and standard deviation (sd) statistics are given for numerical (quantitative) variables. In the comparison of the measurements according to the group, independent groups were used with t/Mann Whitney tests; Comparisons with respect to time were analyzed by repeated ANOVA/Friedman tests, and the relationship between the group and the variables was analyzed using the Chi-square test. $\mathrm{p}$ value $<0.05$ was considered statistically significant.

\section{RESULTS}

There was no statistically significant difference between the groups in terms of gender and age average $(p>0.05)$ (Table 1). There was also no significant difference between the groups in APACHE II scores and comorbidities ( $p>0.05$ ) (Figure 1). The mean age was $72.16 \pm 13.28$ years and the mean APACHE-II score was 18.27 \pm 8.82 .

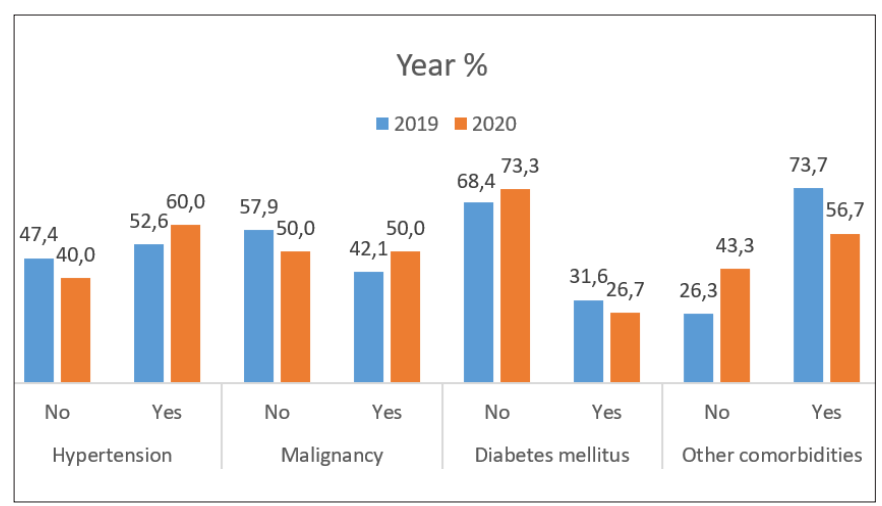

Figure 1. Comorbidities of patients

Table 1. The relationship of groups with gender, age and APACHE II

\begin{tabular}{|lcccc|}
\hline & \multicolumn{2}{c}{ Year } & Total & $\mathbf{p}$ \\
\cline { 2 - 4 } & $\mathbf{2 0 1 9}$ & $\mathbf{2 0 2 0}$ & & \\
\hline Gender & & & & \\
\hline Male & $8(42.1)$ & $16(53.3)$ & $24(49)$ & 0.636 \\
Female & $11(57.9)$ & $14(46.7)$ & $25(51)$ & \\
Age & $69.37 \pm 15.39$ & $73.93 \pm 11.69$ & $72.16 \pm 13.28$ & 0.245 \\
APACHE II & $18.42 \pm 9.51$ & $18.17 \pm 8.52$ & $18.27 \pm 8.82$ & 0.923 \\
\hline
\end{tabular}

Although there was no statistically significant difference between the groups in terms of SOFA score averages, consecutive measurements of SOFA scores were statistically significant. $(\mathrm{p}<0.05)$. Consecutive measurements increased over time in both groups. This increase of consecutive SOFA scores over time did not show a statistically significant difference between the groups. ( $\mathrm{p}>0.05)$ (Figure 2). 


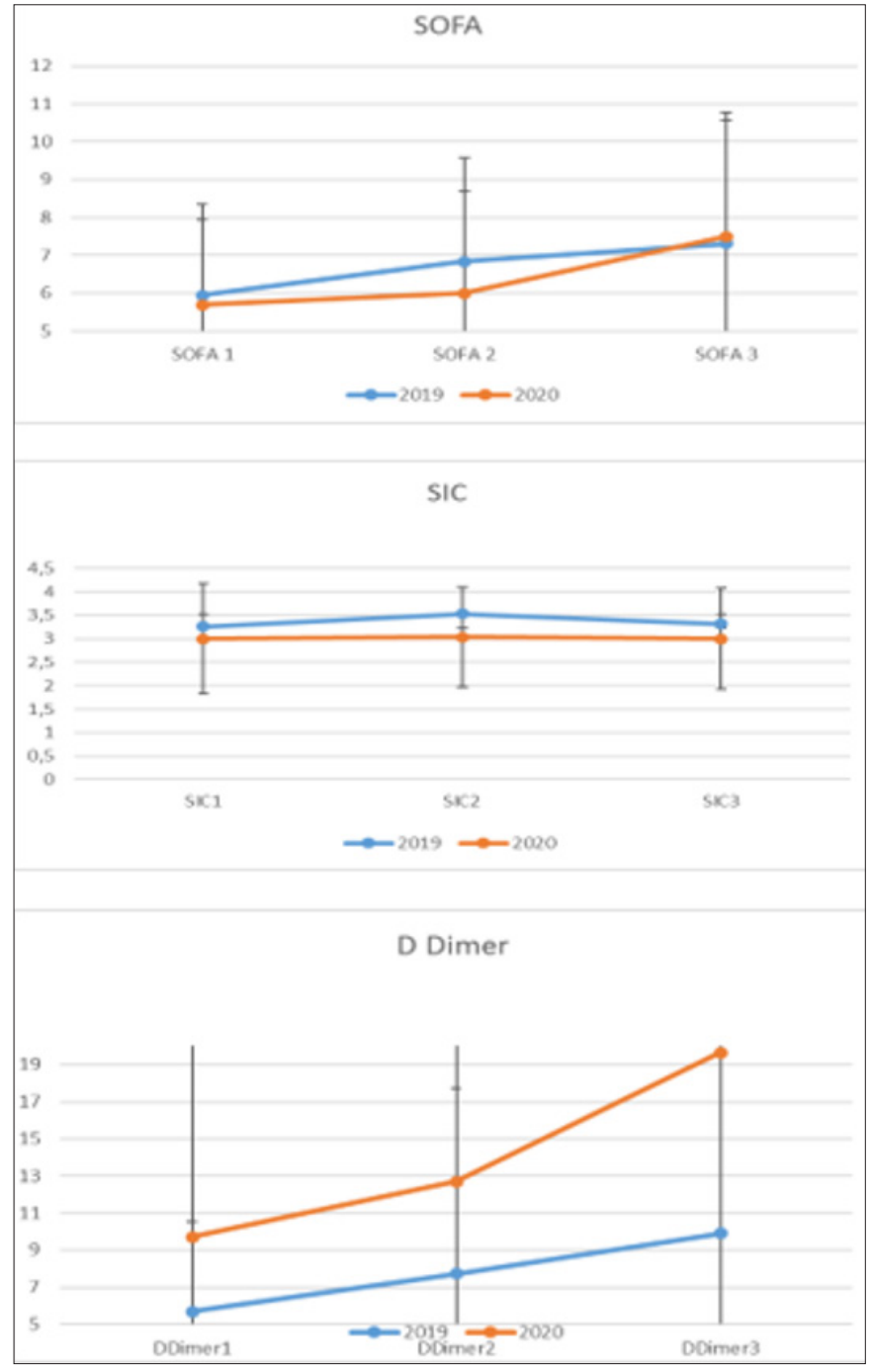

Figure 2. Levels of D-dimer, SOFA and SIC scores by groups

There was no statistically significant difference between the groups in terms of SIC scores ( $p>0.05)$. Consecutive measurements of SIC scores also did not show a statistically significant difference in both groups. ( $p>0.05$ ). The change of SIC scores over time also did not show a statistically significant difference between the groups. ( $\mathrm{p}>0.05$ ) (Table 2).

Table 2. Change of SOFA and SIC measurements over time and the effect of groups

\begin{tabular}{|lcccc} 
& \multicolumn{2}{c}{ Year } & \multirow{2}{*}{ Total } & p \\
\cline { 2 - 3 } & $\mathbf{2 0 1 9}$ & $\mathbf{2 0 2 0}$ & & \\
\hline SOFA 1 & $5.95 \pm 2.01$ & $5.7 \pm 2.67$ & $5.8 \pm 2.42$ & 0.731 \\
SOFA 2 & $6.84 \pm 2.73$ & $6 \pm 2.7$ & $6.33 \pm 2.72$ & 0.296 \\
SOFA 3 & $7.32 \pm 3.25$ & $7.5 \pm 3.28$ & $7.43 \pm 3.23$ & 0.848 \\
SOFA ${ }^{\star}$ Time & & & & $0.000^{\star}$ \\
SOFA ${ }^{\star}$ Year & & & & 0.304 \\
SIC 1 & $3.26 \pm 1.1$ & $3 \pm 1.17$ & $3.1 \pm 1.14$ & 0.437 \\
SIC 2 & $3.53 \pm 1.17$ & $3.03 \pm 1.07$ & $3.22 \pm 1.12$ & 0.136 \\
SIC 3 & $3.32 \pm 1.29$ & $3 \pm 1.08$ & $3.12 \pm 1.17$ & 0.361 \\
SIC ${ }^{*}$ Time & & & & 0.463 \\
SIC ${ }^{*}$ Year & & & & 0.628 \\
\hline
\end{tabular}

There was no statistically significant difference between the groups in terms of the mean D-dimer levels ( $p>0.05)$. However, consecutive measurements of $\mathrm{D}$-dimer levels show a statistically significant difference $(\mathrm{p}<0.05)$. Consecutive measurements have increased over time in both groups. Consecutive D-dimer measurements did not change significantly over time in the 2019 group ( $>0.05)$, while it increased significantly in the 2020 group. $(\mathrm{p}<0.05)$ There was no statistically significant relationship between the d-dimer level and mortality between the groups. ( $\mathrm{p}>0.05)$ (Table 3$)$.

Table 3. Analysis of consecutive measurements of D-dimer levels between groups and their relationship with mortality rate

\begin{tabular}{|lcccc} 
& \multicolumn{2}{c}{ Year } & Total & p \\
\cline { 2 - 4 } & $\mathbf{2 0 1 9}$ & $\mathbf{2 0 2 0}$ & & \\
\hline D-dimer 1 & $5.7 \pm 4.79$ & $9.72 \pm 10.88$ & $8.13 \pm 9.12$ & 0.137 \\
D-dimer 2 & $7.71 \pm 10.03$ & $12.71 \pm 15.2$ & $10.77 \pm 13.54$ & 0.124 \\
D-dimer 3 & $9.91 \pm 13.91$ & $19.65 \pm 20.75$ & $15.87 \pm 18.86$ & 0.078 \\
D-dimer ${ }^{\star}$ Time & & & & 0.002 \\
D-dimer 2019 & & & & 0.268 \\
D-dimer ${ }^{\star} 2020$ & & & & 0.006 \\
Result & & & & 0.418 \\
$\quad$ Ex & $11(57.9)$ & $22(73.3)$ & $33(67.3)$ & \\
\multicolumn{1}{c}{ Discharge } & $8(42.1)$ & $8(26.7)$ & $16(32.7)$ & \\
\hline
\end{tabular}

Analyzing the coagulopathic complications, submassive pulmonary embolism was recorded in one patient in the 2019 group. In the 2020 group, one patient had DIC and one patient had massive pulmonary embolism.

\section{DISCUSSION}

When the patients were analyzed in this study, it was seen that two homogeneous groups were formed similar in terms of age, gender, comorbidity and APACHE scores, and each significant result was very important in terms of showing us the specific differences in COVID-19 pneumonia. When we look at the results, we could not find a significant difference in terms of coagulopathic parameters in the pneumonia groups of the last two years. We are of the opinion that there is no increased coagulopathic process specific to COVID-19 pneumonia.

Autopsy series performed in the early stages of the pandemic had great repercussions all over the world, and the opinion that COVID-19 has high coagulopathic effects has been accepted. In a study by Carsana et al. (10) most of the patients were found to have fibrin thrombus (33/38). D-dimer levels were high in all cases. Likewise, the appearance of capillary microthrombus and pulmonary embolism in the autopsy series conducted by Menter et al. (11) proved once again how important coagulopathy is in the pathogenesis of COVID-19 infection. 
Wichmann et al. (12) reported that there was an unpredictably high level of deep vein thrombosis among the COVID-19 patients who died in their study, and they directly observed massive pulmonary embolism in 33.3\% of the cases. They hypothesized that the main determinant of mortality is coagulopathic complications. In our study, massive pulmonary embolism was developed in one patient in the 2020 group and resulted in mortality. Patients diagnosed with pneumosepsis with similar comorbidities and similar APACHE scores were compared; There was no significant difference in the mortality rate of the patients in the 2020 group compared to the 2019 group.

In a study conducted by Zhou et al. (13) D-dimer levels were found to be significantly higher in patients with mortality in Covid 19 pneumonia, and it was emphasized that it was an independent indicator of in-hospital mortality. It has been hypothesized that the major role in the increase of D-dimer may be due to the increased systemic pro-inflammatory activation triggering the prothrombotic process (14). In a retrospective study by Wang et al. (15) D-dimer levels of 138 patients were shown to be significantly different between patients who required ICU and those who did not $(414 \mathrm{mg} / \mathrm{L}$ vs. 166 $\mathrm{mg} / \mathrm{L})$. They hypothesized that the significant increase in D-dimer was associated with the formation of a large number of microthrombus in the body.

In our study, consecutive measurements of D-dimer levels show a statistically significant difference. Successive measurements have increased over time but there was no statistically significant relationship between the d-dimer level and mortality between the groups.

After studies showing that the addition of anticoagulant therapy to treatment protocols reduces mortality, anticoagulant treatment protocols were routinely applied to each patient, as long as there were no contraindications in our country as in the world. Coagulopathy scores were followed at least every other day in line with the recommendations $(6,16,17)$.

Although the International Thrombosis and Hemostasis Association (ISTH) recommends follow-up with DIC criteria for the diagnosis of DIC, they also stated that these criteria are not suitable for early diagnosis. For this reason, ISTH proposed a new scoring system called "sepsis induced coagulopathy"which is the first scoring system specifically design for coagulation disorders to facilitate early diagnosis and rapid interventions of DIC, and this scoring system has taken its place in the new sepsis 3 definitions $(18,19)$. Sepsis coagulopathy can progress to fatal DIC. Diagnosing DIC makes sepsis management easier and is associated with better outcomes (20). In our study, patients were followed every other day using the SIC score.
Our study has limitations such as the limited number of patients in the groups and its retrospective nature. however, we believe that it will be a pioneer for largescale studies to be carried out in the future.

\section{CONCLUSION}

As a result of the parameters we examined between these two homogeneously formed groups with no significant difference between age and comorbidities, we concluded that the coagulopathic effect of covid pneumonia was not different compared to pneumonias in other years.

\section{ETHICAL DECLARATIONS}

Ethics Committee Approval: The study was carried out with the permission of Eskişehir Osmangazi University Faculty of Medicine Non-Interventional Clinical Researchs Ethics Committee (Date: 16.10.2020, Decision No: 14).

Informed Consent: Because the study was designed retrospectively, no written informed consent form was obtained from patients.

Referee Evaluation Process: Externally peer-reviewed.

Conflict of Interest Statement: The authors have no conflicts of interest to declare.

Financial Disclosure: The authors declared that this study has received no financial support.

Author Contributions: All of the authors declare that they have all participated in the design, execution, and analysis of the paper and that they have approved the final version

\section{REFERENCES}

1. Renu K, Prasanna PL, Valsala Gopalakrishnan A. Coronaviruses pathogenesis, comorbidities and multi-organ damage - a review. Life Sci 2020; 15; 255: 117839.

2. Gavriatopoulou M, Korompoki E, Fotiou D et al. Organ-specific manifestations of COVID-19 infection. Clin Exp Med 2020; 4 : 493-506.

3. Boccia M, Aronne L, Celia B et al. COVID-19 and coagulative axis: review of emerging aspects in a novel disease. Monaldi Arch Chest Dis 2020; 90; 2: 271-6.

4. Connors JM, Levy JH. COVID-19 and its implications for thrombosis and anticoagulation. Blood 2020; 4; 135, 23 : 2033-40.

5. Yıldırım Ö, Bayram M, Özmen RS, et al. Evaluation of hematological indices in terms of COVID-19 related mortality and ICU admission. J Health Sci Med 2021; 4: 5: 666-670.

6. Iba T, Levy JH, Connors JM, Warkentin TE, Thachil J, Levi M. The unique characteristics of COVID-19 coagulopathy. Crit Care 2020; 18; 24, $1: 360: 1-8$.

7. Iba T, Nisio MD, Levy JH, Kitamura N, Thachil J. New criteria for sepsis-induced coagulopathy (SIC) following the revised sepsis definition: a retrospective analysis of a nationwide survey. BMJ Open 2017; 27: e017046: 1-7.

8. Tekyol D, Hökenek NM. Comparison of the ability of the shock index, modified shock index and age shock index to predict mortality in geriatric patients with COVID-19 pneumonia. J Health Sci Med 2021; 4: 5: 634-8. 
9. Iba T, Levy JH, Warkentin TE, Thachil J, van der Poll T, Levi M. Scientific and Standardization Committee on DIC, and the Scientific and Standardization Committee on Perioperative and Critical Care of the International Society on Thrombosis and Haemostasis. Diagnosis and management of sepsis-induced coagulopathy and disseminated intravascular coagulation. J Thromb Haemost 2019; 17: 1989-94.

10. Carsana L, Sonzogni A, Nasr A et al. Pulmonary post-mortem findings in a series of COVID-19 cases from northern Italy: a twocentre descriptive study. Lancet Infect Dis 2020; 20: 1135-40.

11. Menter T, Haslbauer JD, Nienhold R, et al. Postmortem examination of COVID-19 patients reveals diffuse alveolar damage with severe capillary congestion and variegated findings in lungs and other organs suggesting vascular dysfunction. Histopathology 2020; 77: 198-209.

12. Wichmann D, Sperhake JP, Lütgehetmann M, et al. Autopsy findings and venous thromboembolism in patients with COVID-19: a prospective cohort study. Ann Intern Med 2020; 18; 173: 268-77.

13.Zhou F, Yu T, Du R, et al. Clinical course and risk factors for mortality of adult inpatients with COVID-19 in Wuhan, China: a retrospective cohort study. Lancet 2020; 395: 1054-62.

14. Iba T, Connors JM, Levy JH. The coagulopathy, endotheliopathy, and vasculitis of COVID-19. Inflamm Res 2020; 12: 1181-9.

15. Wang $\mathrm{D}, \mathrm{Hu} \mathrm{B}, \mathrm{Hu} \mathrm{C}$, et al. Clinical characteristics of 138 hospitalized patients with 2019 novel coronavirus-infected pneumonia in Wuhan, China. JAMA 2020; 323: 1061-9

16. Tang N, Bai H, Chen X, Gong J, Li D, Sun Z. Anticoagulant treatment is associated with decreased mortality in severe coronavirus disease 2019 patients with coagulopathy. J Thromb Haemost 2020; 5: 1094-9.

17. Rico-Mesa JS, Rosas D, Ahmadian-Tehrani A, White A, Anderson AS, Chilton R. The role of anticoagulation in COVID-19-induced hypercoagulability. Curr Cardiol Rep 2020; 22: 1-6.

18. Iba T, Levy JH, Raj A, Warkentin TE. Advance in the management of sepsis-induced coagulopathy and disseminated intravascular coagulation. J Clin Med 2019; 8: 1-16.

19. Schmitt FCF, Manolov V, Morgenstern J et al. Acute fibrinolysis shutdown occurs early in septic shock and is associated with increased morbidity and mortality: results of an observational pilot study. Ann Intensive Care 2019; 9: 1-15.

20.Inata Y. Should we treat sepsis-induced DIC with anticoagulants? J Intensive Care 2020; 18: 1-3. 